\title{
The Imagination of Divergence in Space
}

\author{
Wenbing Wu, Jinquan Xiong \\ Nanchang Normal University, Nanchang, China \\ Email: wwbysq@finu.edu.cn
}

How to cite this paper: $\mathrm{Wu}, \mathrm{W}$. B., \& Xiong, J. Q. (2021). The Imagination of Divergence in Space. Creative Education, 12, 2863-2868.

https://doi.org/10.4236/ce.2021.1212213

Received: November 7, 2021

Accepted: December 20, 2021

Published: December 23, 2021

Copyright (c) 2021 by author(s) and Scientific Research Publishing Inc. This work is licensed under the Creative Commons Attribution International License (CC BY 4.0).

http://creativecommons.org/licenses/by/4.0/

\begin{abstract}
Divergence is the basis of electromagnetic field theory, and has been a special difficulty in mathematical theory. Understanding these concepts requires strong spatial and abstract thinking. In teaching, through the graphical and quantitative methods, the connotation of divergence itself is perfectly expressed in 1-D, 2-D and 3-D space. Through these methods, the concepts of divergence can be grasped more easily.
\end{abstract}

\section{Keywords}

Divergence, Spatial Thinking, 3-D Space

\section{Introduction}

In the theory of electromagnetic field, in order to simplify the operation, some operator symbols are introduced. They have become indispensable tools in the field theory analysis. The Hamiltonian operator and Laplacian operator are widely used. Hamiltonian operator, the mathematical symbol is $\nabla$. Divergence is a quantity that describes the extent to which air converges from the surrounding area to or from a certain point. The divergence of 3-D space represents the change rate of unit volume of any gas block in unit time. The divergence theorem is the practical application of Gauss's theorem in physics, also called Gauss's divergence theorem, which is often used in vector analysis. The volume fraction of the divergence of the vector field on the volume $\tau$ is equal to the area fraction of the vector field on the closed surface $s$ that defines the volume. The electrostatic field has non-zero divergence and zero curl, which is an active non-rotating field. The divergence of the static magnetic field is zero, and the curl is not zero. It is a rotating passive field. Divergence can represent the rate of change per unit volume of fluid in motion. Simply put, the area where fluid concentrates in motion is convergence, and the area where fluid diverges in motion is divergence. When the divergence value is negative, it is convergent, which is conducive to the de- 
velopment and enhancement of convective weather systems such as cyclones, and when it is positive, it means divergence, which is conducive to the development of weather systems such as anticyclones. Often, the "horizontal divergence" of wind speed is the most widely used in meteorology (Clark, Knops, \& Tilman, 2018; Bano, Niharika, Deepika et al., 2021; Li \& Sun, 2021). Divergence already has been widely used in various aspects of scientific research, such as stochastic models (Pu, Ibrahim, Fu et al., 2021; Gupta \& Haskell, 2020), variational Inequalities (Semenov, 2018; Borkowski \& Jańczak-Borkowska, 2018). Due to the abstract characteristics of divergence, both teachers and students will find it's difficult in the process of teaching and learning the concept: the difficulty of the lecturer lies in how to convey the knowledge contained in these concepts vividly, and how the students grasp the concept completely and accurately. This paper proposes a concrete understanding method for the concept of divergence. This method not only cultivates students' spatial imagination, but also makes the abstract concept of divergence no longer difficult to understand.

\section{Quantitative Understanding of Divergence}

\subsection{Flux and Divergence}

First of all, the theoretical knowledge related to divergence must be listed.

1) Gauss formula:

Let a space closed region $\Omega$ be surrounded by a piecewise smooth closed surface $\Sigma$, and the functions $P(x, y, z), Q(x, y, z)$ and $R(x, y, z)$ have first order continuous partial derivatives on $\Omega$, then there is a formula

$$
\iiint_{\Omega}\left(\frac{\partial P}{\partial x}+\frac{\partial Q}{\partial y}+\frac{\partial R}{\partial z}\right) \mathrm{d} v=\oiint_{\Sigma} P \mathrm{~d} y \mathrm{~d} z+Q \mathrm{~d} z \mathrm{~d} x+R \mathrm{~d} x \mathrm{~d} y
$$

2) Definition of flux

Given a vector field

$$
\boldsymbol{A}(x, y, z)=P(x, y, z) \boldsymbol{i}+Q(x, y, z) \boldsymbol{j}+R(x, y, z) \boldsymbol{k}
$$

The integral of the directed surface $\Sigma$ in the field is:

$$
\Phi=\iint_{\Sigma} \boldsymbol{A} \cdot \mathrm{d} \boldsymbol{S}=\iint_{\Sigma} \boldsymbol{A} \cdot \boldsymbol{n}^{0} \mathrm{~d} S=\iint_{\Sigma} P \mathrm{~d} y \mathrm{~d} z+Q \mathrm{~d} z \mathrm{~d} x+R \mathrm{~d} x \mathrm{~d} y
$$

It's called the flux of the vector field $\mathbf{A}(x, y, z)$ through the directed surface $\Sigma$.

3) Definition of divergence:

Let the vector field $\boldsymbol{A}(x, y, z)$, set a closed surface of the point $M$ in the field, and the area enclosed by $\Sigma$ is $V$. When $V$ shrinks to the point $M$, if the limit $\lim _{V \rightarrow M} \frac{\oiint_{\Sigma} \boldsymbol{A} \cdot \mathrm{d} \boldsymbol{S}}{V}$ exists, the limit value is called the divergence at the point $M$, denoted as $\operatorname{div} \boldsymbol{A}$.

Where 


$$
\operatorname{div} \boldsymbol{A}=\frac{\partial P}{\partial x}+\frac{\partial Q}{\partial y}+\frac{\partial R}{\partial z}
$$

Here introduce Hamilton operator:

$$
\nabla=\frac{\partial}{\partial x} \boldsymbol{i}+\frac{\partial}{\partial x} \boldsymbol{j}+\frac{\partial}{\partial x} \boldsymbol{k}
$$

then:

$$
\operatorname{div} \boldsymbol{A}=\nabla \cdot \boldsymbol{A}
$$

Gauss formula can be expressed as:

$$
\int_{V} \nabla \cdot A \mathrm{~d} v=\oint A \cdot \mathrm{d} s
$$

From the definition of divergence, it describes the density of the flux source. For example, suppose that the thermal radiation intensity vector of each point in space is regarded as a vector field, then the thermal radiation intensity vector around a certain thermal radiation source (such as the sun) all points outward, indicating that the sun is constantly producing new thermal radiation. The source of the divergence is greater than zero. It can also be seen from the definition that divergence is an intensity property of a vector field, just like density, concentration, and temperature, its corresponding extended property is the flux on the surface of a closed area.

From the Gauss flux theorem:

$$
\oint_{s} \boldsymbol{D} \cdot \mathrm{d} \boldsymbol{s}=\sum q
$$

Then the following equation can be obtained:

$$
\nabla \cdot \boldsymbol{D}=\rho
$$

\subsection{Explanation of Divergency}

According to the textbook explanation, divergence is the change rate of power lines volume density, to imagine a charged sphere divergence through spatial imagination in Figure 1. According to the previous analysis results, the true meaning of this sentence can be gotten: electric field divergence is the change rate of power lines to volume at any point in the electric field, which is proportional to the distance between the given point and the charged sphere. This ratio is fixed at any point outside the charged sphere, that is, the number of power lines in a unit volume increases or decreases in proportion to the distance between the volume element and the charged sphere (Figure 2).

Divergence is the representation of the phenomenon that the electric field intensity changes with distance in $3-\mathrm{D}$ space. What about the representation of 1-D and 2-D space? Suppose that A and B are two points in the electric field (Figure 3), The distance of $\mathrm{A}$ and $\mathrm{B}$ from a point charge is $r_{A}, r_{B}$, respectively. As for a point charge, the electric field strength is inversely proportional to the square of the distance: $E=k q / r^{2}$. Then: $E_{A} / E_{B}=r_{B}^{2} / r_{A}^{2}$. This proportional relation is obtained in the linear form, belonging to $1-\mathrm{D}$ space. 


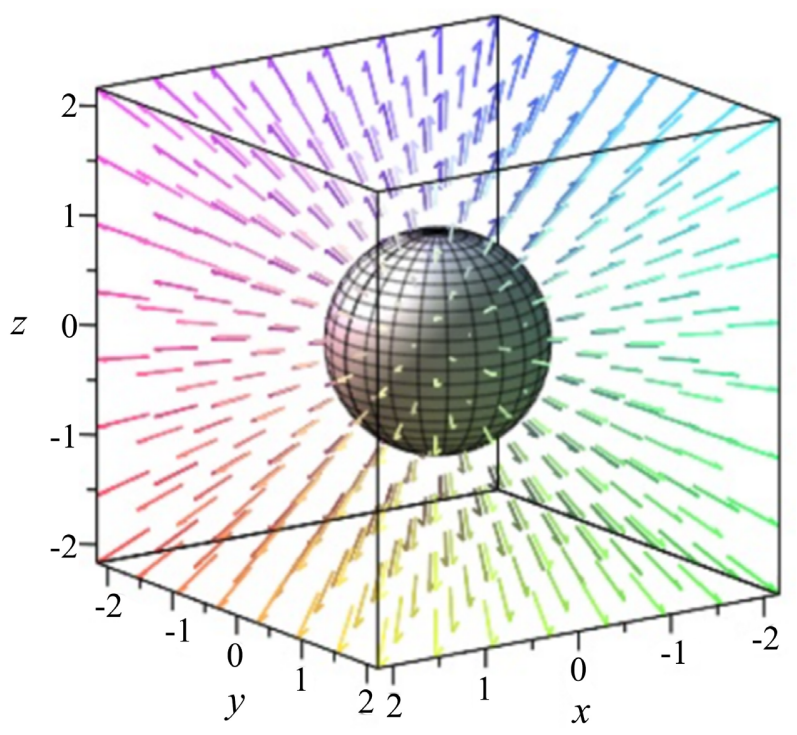

Figure 1. Charged sphere.

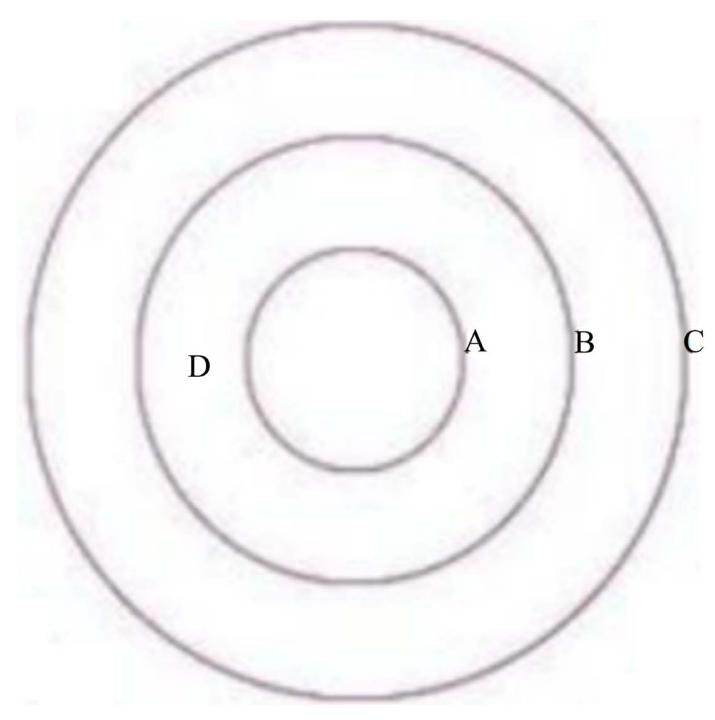

Figure 2. Divergence calculation.

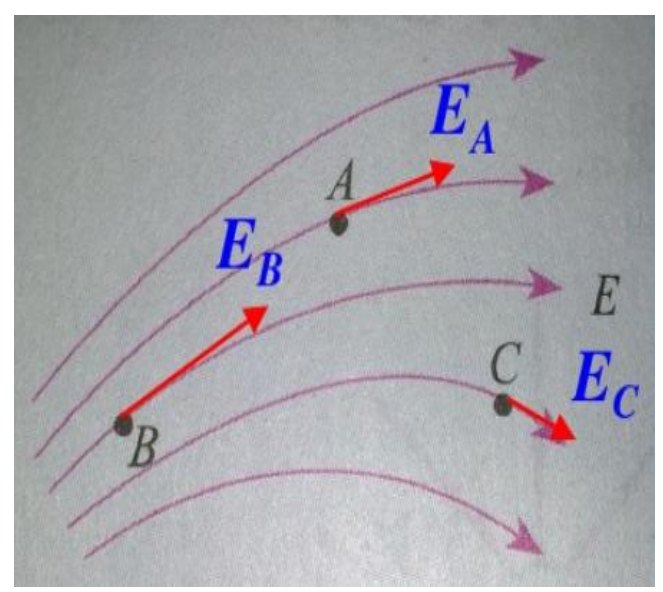

Figure 3. Electric field of point charge. 
Considering the flux problem, assume that a curved surface is also located in the electric field of one point charge (Figure 4).

With the concept of electric field intensity, flux and divergence, now we imagine a scene as follows: holding a piece of glass in your left hand and a glass ball in your right hand, you leave the light bulb in front of you at a constant speed, and at the same time, your eyes are staring at one light emitted by the bulb (Figure 5), then the light in front of you, the light passing through the piece of glass and the glass ball, the lines all weaken or decrease proportionally with the distance as you are away from the bulb, and the meaning of the above three scenes is exactly the same. In this action, combined with Figure 1, Figure 4 and Figure 5, the fact that the electric field intensity changes with the distance is perfectly expressed in 1-D, 2-D and 3-D space. It is Gauss formula that combines 2-D space with 3-D space:

$$
\iiint_{\Omega}\left(\frac{\partial P}{\partial x}+\frac{\partial Q}{\partial y}+\frac{\partial R}{\partial z}\right) \mathrm{d} v=\oiint_{\Sigma} P \mathrm{~d} y \mathrm{~d} z+Q \mathrm{~d} z \mathrm{~d} x+R \mathrm{~d} x \mathrm{~d} y
$$

Through the above analysis, we can get the essential connotation of the divergence symbol itself, that is, the divergence represents the change rate of the number of power lines contained in one unit volume in the field, and this number will change with the distance of the unit volume from the charged sphere.

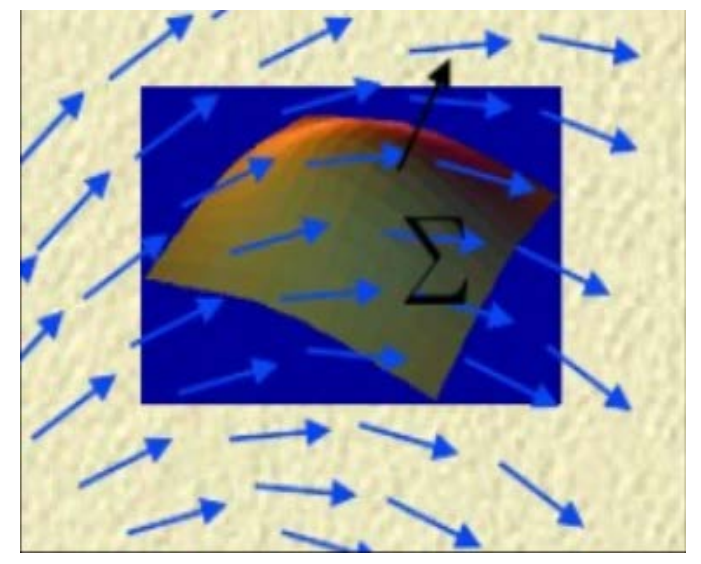

Figure 4. Flux map.
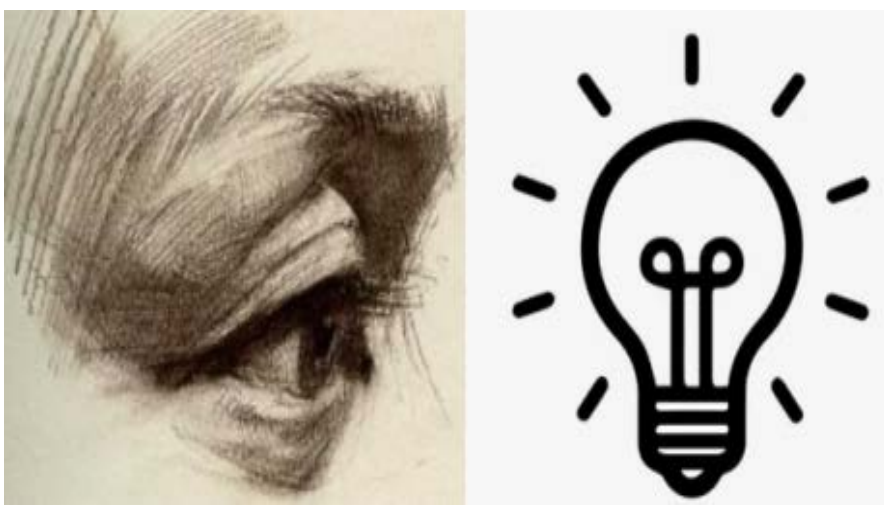

Figure 5. 1-D space interpretation of divergence. 


\section{Conclusion}

Through spatial imagination, the connotation of divergence is deeply analyzed: when imaging the action that you hold a piece of glass in your hand, the electric field intensity changes with the distance is perfectly expressed in 1-D, 2-D and 3-D space, so the concepts of divergence become easily grasped for the students.

\section{Funding}

This paper is supported by Research Foundation of the Nanchang Normal University for Doctors (NSBSJJ2018014). Research project of major education and teaching reform in Fujian Province (FBJG20190284).

\section{Conflicts of Interest}

The authors declare no conflicts of interest regarding the publication of this paper.

\section{References}

Bano, S., Niharika, G. L., Deepika, T. et al. (2021). Weather Divergence of Season through Regression Analytics. Intelligent Sustainable Systems, 119-129. https://doi.org/10.1007/978-981-16-2422-3 10

Borkowski, D., \& Jańczak-Borkowska, K. (2018). Backward Stochastic Variational Inequalities Driven by Multidimensional Fractional Brownian Motion. Opuscula Mathematica, 38, 307. https://doi.org/10.7494/OpMath.2018.38.3.307

Clark, A. T., Knops, J. M. H., \& Tilman, D. (2018). Contingent Factors Explain Average Divergence in Functional Composition over 88 Years of Old Field Succession. Journal of Ecology, 107, 545-558. https://doi.org/10.1111/1365-2745.13070

Gupta, A., \& Haskell, W. B. (2020). Convergence of Recursive Stochastic Algorithms Using Wasserstein Divergence.

Li, Y., \& Sun, Y. (2021). A Multi-Site Stochastic Weather Generator for High-Frequency Precipitation Using Censored Skew-Symmetric Distribution. Spatial Statistics, 41, 100474. https://doi.org/10.1016/j.spasta.2020.100474

$\mathrm{Pu}, \mathrm{W}$., Ibrahim, S., Fu, X. et al. (2021). Fiber-Sampled Stochastic Mirror Descent for Tensor Decomposition with $\beta$-Divergence. 2021 IEEE International Conference on Acoustics, Speech and Signal Processing (ICASSP), Toronto, 6-11 June 2021. https://doi.org/10.1109/ICASSP39728.2021.9413830

Semenov, V. V. (2018). Modified Extragradient Method with Bregman Divergence for Variational Inequalities. Journal of Automation and Information Sciences, 50, 26-37. https://doi.org/10.1615/JAutomatInfScien.v50.i8.30 